\title{
Lactobacillus gasseri
}

National Cancer Institute

\section{Source}

National Cancer Institute. Lactobacillus gasseri. NCI Thesaurus. Code C123492.

A species of Gram-positive, rod shaped, anaerobic bacteria in the phylum Firmicutes. This species is nonmotile and non spore forming. L. gasseri is found in the gastrointestinal tract and the vaginal tract where it produces lactic acid by fermentation; it also produces hydrogen peroxide. It is a causative agent of Fournier's gangrene. 